\title{
Cloroquina e os efeitos adversos da atual conjuntura política
}

\author{
Chloroquine and the adverse events of the current political situation
}

Cloroquina y los efectos adversos de la actual coyuntura política

Recebido: 17 jun 2020

Revisado: 8 set 2020

Aceito: 14 set 2020

Autor de correspondência:

Samara Jamile Mendes

samarajm@gmail.com

Conflito de interesses: Os autores declaram não haver nenhum interesse profissional ou pessoal que possa gerar conflito de interesses em relação a este manuscrito.

\section{Samara Jamile MENDES ${ }^{(1)}$ \\ Vivian Cristina Borges ZANHOLO ${ }^{(2)}$}

\author{
${ }^{(1)}$ Universidade de São Paulo - USP, Faculdade de Ciências Farmacêuticas - FCF, São Paulo, SP, \\ Brasil. \\ (2)Faculdade Oswaldo Cruz - FOC, São Paulo, SP, Brasil.
}

\section{Resumo}

Em um cenário tão delicado e sensível da pandemia de COVID-19, os desafios enfrentados não se restringem somente ao combate do vírus, mas também às crises econômica e político-social por quais os países atravessam. Considerando esse contexto crítico por qual estamos passando, tem-se como principal objetivo deste artigo de debate: a análise e a reflexão sobre o tema, observando-se, a partir do cenário brasileiro: o uso indiscriminado da cloroquina e como ele se relaciona à atual conjuntura política do país. Enquanto empresas farmacêuticas intensificam pesquisas e estudos clínicos em busca de vacinas e medicamentos eficazes na prevenção e tratamento do coronavírus, alguns governos disseminam e impõem a prescrição da cloroquina/hidroxicloriquina como sendo a cura para a pandemia, mesmo sem nenhuma comprovação de segurança e eficácia para essa indicação. No Brasil, temos um governo ultraliberal e neofascista que desde o início da pandemia vem tomando ações contrárias as orientadas pela OMS. Como agravante, possui ainda um cenário bem peculiar como: troca de dois ministros da saúde nos últimos 3 meses, incorporação da cloroquina no protocolo de tratamento de pessoas infectadas por COVID19 e importação de 2 milhões de doses do medicamento dos Estados Unidos. Neste contexto, cabe aos trabalhadores da saúde e cientistas um olhar crítico sobre as medidas de enfrentamento à pandemia e suas contradições ao modo de produção capitalista.

Descritores: Pandemias; Infecções por Coronavirus; Política; Brasil.

\section{Abstract}

In a delicate and sensible scenario of the COVID-19 pandemic, the challenges faced are not restricted only to oppose the virus, but also to the economic and political-social crises that countries are going through. Considering this critical context that we are going through, the main objective of this debate article is the analysis and reflection on the theme, observing, from the Brazilian scenario: the indiscriminate use of chloroquine and how it relates to current political situation in the country. While pharmaceutical companies intensify research and clinical studies in search of vaccines and drugs effective in the prevention and treatment of coronavirus, some governments disseminate and impose the prescription of chloroquine / hydroxychloriquin as a cure for the pandemic, even without any proof of safety and efficacy for that indication. In the Brazilian sphere, we have an ultraliberal and neo-fascist government that, since the beginning of the pandemic, has been taking actions contrary to those prescribed by WHO. In Brazil, it has a very peculiar scenario such as: changing two ministers of health in the last 3 months, incorporating chloroquine in the treatment protocol for people infected with COVID-19 and importing 2 million doses of the drug from the United States. In this context, it is up to health workers and scientists to be critical at measures to deal with the pandemic and its contradictions to the capitalist mode of production.

Keywords: Pandemics; Coronavirus Infections; Politics; Brazil. 


\section{Resumen}

En un escenario delicado y sensible de la pandemia de COVID-19, los desafíos que se enfrentan no se limitan solo a combatir el virus, sino también a las crisis económicas y político-sociales que atraviesan los países. Teniendo en cuenta este contexto crítico que estamos atravesando, el objetivo principal de este artículo del debate es: el análisis y la reflexión sobre el tema, observando, desde el escenario brasileño: el uso indiscriminado de la cloroquina y cómo se relaciona con la situación política actual en el país. Mientras las compañías farmacéuticas intensifican la investigación y los estudios clínicos en busca de vacunas y medicamentos eficaces en la prevención y el tratamiento del coronavirus, algunos gobiernos difunden e imponen la prescripción de cloroquina/hidroxicloriquina cómo una cura para la pandemia, incluso sin ninguna evidencia de seguridad y eficacia por esa indicación. En la esfera brasileña, tenemos un gobierno ultraliberal y neofascista que, desde el principio de la pandemia, ha estado tomando medidas contrarias a las prescritas por la OMS. En Brasil, tiene un escenario muy peculiar como: cambiar dos ministros de salud en los últimos 3 meses, incorporar cloroquina en el protocolo de tratamiento para personas infectadas con COVID-19 e importar 2 millones de dosis de la droga de los Estados Unidos. En este contexto, corresponde a los trabajadores de la salud y los científicos analizar críticamente las medidas para hacer frente a la pandemia y sus contradicciones con el modo de producción capitalista.

Palabras-claves: Pandemias; Infecciones por Coronavirus; Política; Brasil.

\section{Introdução}

Não há como negar que a pandemia de COVID-19 é o um dos temas (se não o) mais discutido atualmente. Na data de 6 de junho de 2020, só o Brasil contabilizou 614.941 casos confirmados (9\% dos 6.797 .633 globais), sendo 34.021 números de óbitos (8,5\% de 396.388 globais). ${ }^{1}$ O número, além de crescente, não possui previsão de declínio em um futuro próximo.

Nesse cenário tão crítico, enquanto empresas farmacêuticas realizam estudos e pesquisas para descobertas de vacinas e medicamentos que possam prevenir ou tratar pacientes infectados, os governos dos países direcionam esforços em medidas alternativas para tentar controlar a disseminação do vírus e diminuir a quantidade de mortes diárias.

Na busca incessante das soluções e curas para a crise sanitária, surge a suposta promessa de cura com a utilização do medicamento cloroquina, medicamentos utilizados para agravos bem delimitados pelos protocolos clínicos. Neste contexto fazse necessário algumas reflexões sobre a possibilidade de utilização da cloroquina como profilaxia/tratamento, considerando que ainda não há nenhuma comprovação científica e nem tampouco clínica de suas segurança e eficácia. Além de compreender as delimitações e influência do Estado brasileiro neste momento sobre o negacionismo da ciência.

Neste contexto, este artigo tem por objetivo um debate que venha fazer apontamentos sobre a o uso do medicamento cloroquina para o Covid-19 e seus desdobramentos políticos neofascistas da conjuntura brasileira. 


\section{Cloroquina no cenário regulatório brasileiro}

O desenvolvimento de um medicamento novo passa por algumas etapas até que possa ser utilizado como escolha terapêutica como: a descoberta da nova molécula, o desenvolvimento e a pesquisa do novo fármaco e finalmente o registro no órgão regulador. Esse processo completo pode levar até 14 anos para ser finalizado. ${ }^{2}$

No caso dos medicamentos à base de cloroquina ou hidroxicloroquina, já registrados nos país, as indicações atuais aprovadas são as seguintes: afecções reumáticas e dermatológicas; artrite reumatoide ou reumatoide juvenil; lupus eritematoso sistêmico ou discoide; condições dermatológicas provocadas ou agravadas pela luz solar; malária; tratamento das crises agudas e tratamento supressivo de malária por Plasmodium vivax, $P$. ovale, $P$. malária e cepas sensíveis de $P$. falciparum. ${ }^{3}$

Para que esses medicamentos possam ser utilizados no tratamento de Covid-19, é necessário solicitar à Agência Nacional de Vigilância Sanitária - ANVISA a inclusão de uma nova indicação terapêutica na bula, cujo processo somente poderá ser deferido após comprovação de segurança e eficácia por meio de estudos clínicos com número representativo de participantes. ${ }^{4}$

Apesar da ANVISA já ter aprovado o início de ensaios clínicos nos país, ainda não existe nenhum estudo concluído que comprove os benefícios da hidroxicloroquina no tratamento da Covid-19. ${ }^{4}$

Adicionalmente, vale enfatizar que a ANVISA possui um Plano de Contingência COVID-19, com gestão de riscos corporativos e está tomando medidas para auxiliar nesse momento crítico da pandemia como: flexibilização e simplificação de regulamentos, aconselhamento, orientação e assistência técnicas. Todos os produtos para saúde e medicamentos relacionados ao diagnóstico e tratamento do COVID-19 estão sendo priorizados nas análises. $^{5}$

É importante pontuar que (difosfato de) cloroquina e (sulfato de) hidroxicloroquina estão padronizados para acesso no Sistema Único de Saúde - SUS, por meio dos Componentes Estratégico e Especializados, respectivamente. ${ }^{6}$

Após a divulgação em meios de comunicação de que a cloroquina poderia ser utilizada como tratamento ao COVID-19, em meados do mês de março de 2020, houve um aumento de uso indiscriminado do medicamento.

O desabastecimento do fármaco foi um risco inerente ocorrido. Além disso, os riscos ligados a automedicação e neste caso, o uso off label, ${ }^{7}$ são catalogados pelo IBM Micromedex Drug, sendo eles: diarreia e gastrite; alterações cutâneas; alterações visuais, 
incluindo visão embaçada e visão noturna prejudicada; dificuldade em ouvir e tocar nos ouvidos. $^{8}$

Para evitar o desabastecimento do mercado, que poderia prejudicar inclusive pacientes que utilizam o medicamento para tratamento de doenças crônicas, a ANVISA publicou as Resoluções RDC n. 351/2020 e RDC n. 354/2020, que diz que os medicamentos que possuem cloroquina ou hidroxicloroquina passam a ser comercializados somente com "Receita de Controle Especial". Essa mudança visou garantir o acesso para as pessoas que estejam fazendo tratamento somente para as atuais indicações aprovadas.

\section{A cloroquina como estratégia neofascista}

O Brasil neste momento, além da pandemia, passa por um governo autoritário, ultraliberal, advindo de um movimento chamado de "Bolsonarista". Este governo utiliza de uma construção midiática falsa sobre o uso da cloroquina e a busca por medicamentos que possam curar a infecção causada pelo COVID-19. Entretanto as preocupações são com os efeitos adversos advindos deste uso indiscriminado e com a mesma intensidade sobre o negacionismo científico com a atual conjuntura neofacistizante, compreendendo que Bolsonaro expressa características como: misoginia, racismo e o nacionalismo submisso à influência dos EUA nos países da América do Sul. 9,10

Ainda, como já descrito por Roberts ${ }^{11}$ em "The Long Depression", em momento de longa depressão do capitalismo, recuperar o lucro requer atitudes ultraliberais. É neste cenário que se vê o avanço das forças sociais conservadoras (e neofascistas) na tentativa de reaquecer a acumulação e 'animar' as projeções de mercados. ${ }^{12}$

A exploração das grandes corporações e monopólios da indústria farmacêutica no campo saúde e a concentração de mercado é gravíssima em países da América Latina, especialmente pelos processos de desenvolvimento tecnológico, de inovação de medicamentos e na falta de estratégias de proteção da propriedade industrial. ${ }^{13,14}$

O processo de desenvolvimento tecnológico de medicamentos resulta das atividades de pesquisa básica (mais arriscadas e subsidiadas com financiamento público) e da medicina translacional (alinhamento e transcrição do conhecimento científico em tecnologia), que são realizadas nas universidades e centros de pesquisa, articuladas às atividades de pesquisa e desenvolvimento interna das empresas. ${ }^{13}$

Por isso, ciência e política não são elementos separados, mas é preciso situar a ciência positivista que é utilizada nas ciências naturais, como os estudos clínicos de medicamentos e porque também não nas ciências sociais e humanas, que acabam por ter um 
distanciamento e causando o entendimento de que fazer ciência está apenas ligada ao que é tecnológico e de áreas mais duras, sem uma compreensão do social. ${ }^{15}$

Para a compreensão da totalidade em aspectos de saúde pública, Jaime Breilh ${ }^{15}$, sanitarista e pesquisador equatoriano, faz uma crítica a epidemiologia tradicional ou clássica e os caminhos para os chamados determinantes sociais da saúde, especialmente àqueles apenas fundantes em fatores de risco e causalismo, calcado em noções positivistas, que reduz ao plano dos fenômenos individuais ou um somatório de frações tendo como objeto central cada indivíduo afetado por fatores de risco. A ideia de determinantes está ancorada no 'determinismo', que afirma que um evento qualquer vem de uma causa, e que dada a causa aceita, o evento decorrerá dela, invariavelmente. ${ }^{16}$

Como proposta, Breilh ${ }^{15}$ apresenta a epidemiologia crítica que diz respeito ao conjunto de paradigmas compondo o modelo praxiológico (realismo dialético), e ao mesmo tempo rompendo com um modelo indutivo empirista baseado na correlação pontual de fatores observados experimentalmente, também distancia-se de uma metodologia limitada ao subjetivismo, que não diz sobre as relações, contradições e conflitos entre grupos antagônicos em esferas de poder e equidade, que fica reduzida a concepções culturais fragmentadas e interesses ou perspectivas individuais. ${ }^{16}$

Breilh ${ }^{15}$ apresenta em sua concepção de determinação, a ideia de 'processos determinados' aqueles que têm um modo definido de devir, ou seja, para não cair no determinismo (unicausal), a explicação deve se relacionar com o 'modo de devir', forma, ato ou processo em que um objeto adquire suas propriedades. E para a saúde não seria diferente. É preciso compreender determinação da saúde com determinantes não causais, mas de processos históricos que geram os problemas de saúde coletiva.

\section{Considerações finais}

Não há como não ponderar que os tempos que vivemos no Brasil, com um governo neofascista, ${ }^{9}$ em que no meio de uma pandemia, cujo número de óbitos ultrapassa $42.720,{ }^{1}$ as duas modificações de Ministros da Saúde nos últimos três meses, geraram ainda mais instabilidade ao governo.

Além disso, no final do mês de maio, foi publicado, às pressas e de forma autoritária pelo MS, um protocolo para o uso da cloroquina, inclusive em espaço ambulatorial, como por exemplo na Atenção Básica. Protocolo esse que abrange todos os casos clínicos de infecção por COVID-19, incluindo os mais leves.

Não é nenhuma novidade que o SUS tem em sua estruturação os princípios de municipalização e autonomia decisória aos Estados e Municípios, o que permite a estes 
entes uma possibilidade de não utilizar o protocolo. Porém, em um país historicamente invadido pela ofensiva neoliberal, pelo poder da mídia enquanto instrumento de desinformação em saúde, o poder do complexo médico-industrial que sempre envolveu todas as possibilidades de desenvolver um sistema de saúde partindo da promoção da saúde e de olhar criticamente para a determinação social do processo saúde-doença, um medicamento neste momento, tem um símbolo de salvação impactante na vida da população brasileira, tornando sua utilização quase que compulsória.

Vale ainda ressaltar que, até o presente momento, não houve nenhum estudo publicado que comprovem os benefícios da utilização da cloroquina no tratamento de COVID-19. Ao contrário, os estudos demonstraram aumento da mortalidade pelo COVID-19 e de arritmia, sugerindo que o uso de cloroquina e/ou hidroxicloroquina (com ou sem associação de outros medicamentos) não deva ter indicação para o tratamento do COVID19 e que são necessários novos estudos, principalmente com ensaios clínicos randomizados. ${ }^{17,18}$

Ainda no meio da pandemia, mesmo após a Organização Mundial da Saúde - OMS ter decretado a suspensão de testes dos medicamentos para pacientes com COVID-19, considerando o aumento de risco de morte com a utilização dos mesmos, os EUA enviaram 2 milhões de doses de cloroquina ao Brasil, demonstrando claramente interesses econômicos na imposição da utilização do mesmo no país. ${ }^{19}$

Após todos os fatos e apontamentos descritos anteriormente, e considerando o atual cenário da conjuntura política neofascista no país, cabe a nós, trabalhadores da saúde e cientistas, trazer à tona, com responsabilidade não só técnica, mas político-social, um olhar crítico sobre as aparências das medidas de enfrentamento à pandemia e suas contradições ao modo de produção capitalista.

\section{Referências}

1. Coronavirus Resource Cente [homepage]. [Baltimore, MD: 2020 citado 6 jun. 2020]. Disponível em: https://coronavirus.jhu.edu/map.html.

2. Devalapally $\mathrm{H}$, Chakilam $\mathrm{A}, \mathrm{Amiji}$ MM. Role of nanotechnology in pharmaceutical product development. J Pharm Sci. 2007;96(10):2547-65. http://dx.doi.org/10.1002/jps.20875.

3. Sociedade Brasileira de Farmácia Clínica. Posicionamento: uso de hidroxicloroquina e cloroquina para o COVID-19. [Brasília, DF]: SBFC, 2020 Citado 29 out. 2020]. Disponível em: http://www.farmaciaclinica.org.br/wp-content/uploads/2020/03/PosicionamentoSBFC-USO-DE-HIDROXICLOROQUINA-E-CLOROQUINA-PARA-COVID-19.pdf. 
4. Agência Nacional de Vigilância Sanitária (BR). Esclarecimentos sobre hidroxicloroquina e cloroquina. [Brasília, DF]: 19 mar. 2020, atualizado 30 mar. 2020 citado 29 out. 2020. Disponível em: http://antigo.anvisa.gov.br/resultado-de-

busca?p_p_id $=101 \&$ p_p_lifecycle $=0 \&$ p_p_state $=$ maximized \&p_p_mode $=$ view\&p_p_col_id $=$ column1\&p_p_col_count=1\&_101_struts_action=\%2Fasset_publisher\%2Fview_content \&_101_assetEntryId= $5817064 \&$ 101_type $=$ content\&_101_groupId=219201\&_101_urlTitle=covid-19-esclarecimentossobre-hidroxicloroquina-ecloroquina\&redirect=http\%3A\%2F\%2Fantigo.anvisa.gov.br\%2Fresultado-debusca\%3Fp_p_id\%3D3\%26p_p_lifecycle\%3D0\%26p_p_state\%3Dnormal\%26p_p_mode\%3Dview\%26 p_p_col_id\%3Dcolumn-

1\%26p_p_col_count\%3D1\%26_3_groupId\%3D0\%26_3_keywords\%3Dcloroquina\%26_3_cur\%3D1\%2 6_3_struts_action\%3D\%252Fsearch\%252Fsearch\%26_3_format\%3D\%26_3_formDate\%3D144182447 6958\&inheritRedirect=true.

5. Agência Nacional de Vigilância Sanitária (BR). Regulamentos. [Brasília, DF]: 21 set. 2020, atualizado 29 out. 2020. Disponível em: https://www.gov.br/anvisa/ptbr/assuntos/paf/coronavirus/regulamentos-e-medidas, acessado em 29/10/2020

6. Ministério da Saúde (BR). Departamento de Assistência Farmacêutica e Insumos Estratégicos. Relação Nacional de Medicamentos Essenciais: Rename 2020 [recurso eletrônico]. Brasília, DF: Ministério da Saúde, 2020.

7. Ministério da Saúde (BR). Uso off label: erro ou necessidade?. Rev Saude Publica. 2012;46(2):395-7. http://dx.doi.org/10.1590/S0034-89102012000200026. vol.46 no.2 São Paulo. 2012.

8. IBM Corporation. IBM Micromedex Drug Info por Truven Health Analytics Inc. c2020 [citado 19 mar. 2020]. Disponível em: http://www.micromedexsolutions.com/micromedex2/librarian.

9. Bray M. Antifa. O manual antifacista. São Paulo: Autonomia Literária; 2019.

10. Konder L. Introdução ao facismo. Rio de Janeiro: Edições do Graal; 1977.

11. Roberts M. The long depression: how it happened, why it happened, and what happens next. Chicago: Haymarket Books; 2016.

12. Mendes À, Carnut L. Lucro ou vidas? Coronavírus e o voto de Minerva. Rev Mov. 6 abr. 2020 [citado 4 maio 2020]. Disponível em https://movimentorevista.com.br/2020/04/lucroou-vidas-coronavirus-e-o-voto-de-minerva/. 
13. Kaitin K. Deconstructing the drug development process: the new face of innovation. Clin Pharmacol Ther. 2010:87(3):356- 61. http://dx.doi.org/10.1038/clpt.2009.293.

14. Gadelha CAG, Temporão JG. Desenvolvimento, inovação e saúde: a perspectiva teórica e política do complexo econômico-industrial da saúde. Cienc Saude Colet. 2018; 23(6):1891902. https://doi.org/10.1590/1413-81232018236.06482018.

15. Breilh J. Epidemiologia crítica: ciência emancipadora e interculturalidade. Rio de Janeiro: FIOCRUZ; 2006.

16. Souza IFP, Mendes Á, Carnut, L. História política e pensamento epidemiológico: Breilh e a economia política da saúde. Rev Guillermo de Ockham. 2019;17(1):77-84. https://doi.org/10.21500/22563202.4039.

17. Gragnani J. Coronavírus: o que dizem os estudos publicados sobre cloroquina, defendida por Bolsonaro e Trump, 19 maio 2020 [citado 6 jun. 2020]. Disponível em: https://www.bbc.com/portuguese/brasil-52686122.

18. Sanar Medicina. Pesquisas científicas sobre o uso de cloroquina e hidroxicloroquina na Covid-19. [abr 2020 citado 6 de jun 2020]]. Disponível em: https://www.sanarmed.com/pesquisas-cientificas-sobre-uso-hidroxicloroquina-covid-19.

19. EUA enviam dois milhões de doses de hidroxicloroquina ao Brasil para tratar a Covid -19. Carta Capital. 1 jun. 2020 [citado 15 jun. 2020]. Disponível em: https://www.cartacapital.com.br/mundo/eua-enviam-dois-milhoes-de-doses-de-hidroxicloroquinaao-brasil-para-tratar-a-covid-19/?utm_campaign=novo_layout_newsletter__0162020\&utm_medium=email\&utm_source $=R D+$ Station. 
Samara Jamile Mendes | ORCiD: 0000-0003-3107-8233

Possui graduação em Farmácia pela Universidade Federal de Santa Catarina (2011) e mestrado em Farmácia pela Universidade Federal de Santa Catarina (2013). Doutorado em Ciências, pela Faculdade de Ciências Farmacêuticas da USP/SP (2020), desenvolvendo tese com estudo etnográfico em Serviços Farmacêuticos na Atenção Primária à Saúde. Foi membro da equipe técnica-pedagógica do Curso de Gestão da Assistência Farmacêutica - UFSC (2013-2016). Atua na Diretoria de Assistência Farmacêutica na Secretaria de Estado de Saúde de São Paulo, Brasil.

Vivian Cristina Borges Zanholo | ORCiD: 0000-0002-1207-9460

Formada em Farmácia-bioquímica pela Faculdade de Ciências Farmacêuticas da Universidade de São Paulo (2009), pós-graduada em Gestão de Negócios com ênfase no Mercado Farmacêutico pela Escola Superior de Propaganda e Marketing - ESPM (2015) e em Direto Sanitário pelas Faculdades Oswaldo Cruz - FOC (2018). Atua com Coordenadora de Assuntos Regulatórios em Indústria Farmacêutica, possuindo 14 anos de experiência na área. 